\title{
Mathematical Model for 802.15.4 Networks in NS2: Performance Analysis with BO and SO
}

\author{
Vinamrata \\ Dept. of Electronics \& Comm. \\ SSET, SHIATS \\ Allahabad, U.P., India
}

\author{
Ajit Anupam \\ Dept. of Electronics \& Comm. \\ D. Y. Patil College of Engg. \\ Pune, Maharastra, India
}

\author{
Amit Anupam \\ Dept. of Electronics \& Comm. \\ D. Y. Patil College of Engg. \\ Pune, Maharastra, India
}

\begin{abstract}
Network Simulator (NS) is a discrete event simulator targeted at networking research that provides substantial support for simulation of various networks. Performance evaluation in effective manner is the main concern of this paper. This paper, presents a mathematical model to work with the presimulation TCL file and post-simulation trace file evaluation for the 802.15.4 networks. The impact of $\mathrm{BO}$ and $\mathrm{SO}$ on performance of 802.15 .4 with varying duty cycle is analyzed considering various parameters like packet delivery ratio, average end-to-end delay and energy consumption in different state: receiving, transmitting and idle mode.
\end{abstract}

\section{Keywords}

NS2, MAC, CAP, CFP, GTS, 802.15.4

\section{INTRODUCTION}

The IEEE 802.15.4 standard has been designed to specify the physical layer (PHY) and medium access control (MAC) sublayer for low power consumption, short range and low-rate wireless personal area network (LR-WPAN) [1]. The IEEE 802.15.4 standard defines the protocols and interconnection of devices via radio communication that uses CSMA/CA medium access mechanism and can operate in beacon-enabled mode and non-beacon enabled mode and has three types of topologies: star; peer-to-peer; and cluster tree topologies. In a LR-WPAN network, two types of devices are used: full function device (FFD) and reduced function device (RFD). FFD is a fully functional device which can be a PAN coordinator, a network coordinator, or just a device that periodically sends the beacon frames to the device nodes and provide synchronization services in an established network. RFD is a end device with reduced functionality, that cannot communicate with any other device in addition to coordinator. IEEE 802.15.4 can operate in three licensed free industrial scientific medical (ISM) frequency bands. These are having different data rates over different frequency. $250 \mathrm{kbps}$ (or expressed in symbols, $62.5 \mathrm{ksym} / \mathrm{sec}$ ) in the $2.4 \mathrm{GHz}$ band, 40 kbps $(40 \mathrm{ksym} / \mathrm{sec})$ in the $915 \mathrm{MHz}$ band, and $20 \mathrm{kbps}$ in the 868MHz. Total 27 channels are allocated in 802.15.4, with 16 channels in the $2.4 \mathrm{GHz}$ band, 10 channels in the $915 \mathrm{MHz}$ band, and 1 channel in the $868 \mathrm{MHz}$ band. A device in an 802.15.4 network can use either a 64-bit IEEE address or a 16-bit short address or a 16-bit short address assigned during association procedure, and a single 802.15.4 network can accommodate upto $64 \mathrm{k}\left(2^{16}\right)$ devices.

IEEE 802.15.4 MAC supports not only contention based mechanism in contention access period (CAP) but also guaranteed time slot (GTS) in contention free period (CFP) scheme under beacon-enabled mode. The challenge of the 802.15.4 beacon enabled mode is to improve throughput and bandwidth utilization in CAP, and CFP, respectively. The length of CFP allocation period may increase as the number of requested CFP slots increases, but the length of CAP used by the devices will be decreased.

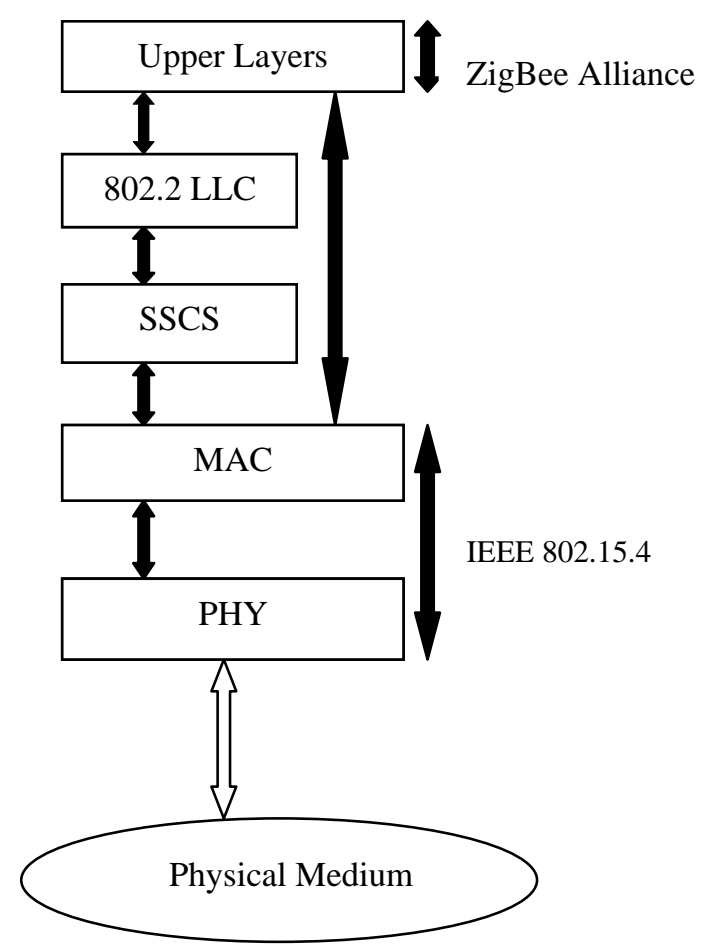

Figure 1 IEEE 802.15.4 for NS2 Simulator

The 802.15.4 NS2 [2] simulator developed at the Joint Lab of Samsung and the City University of New York confirms to IEEE P802.15.4/D18 Draft. The IEEE 802.15.4 working group focuses on the standardization of the bottom two layers of ISO/OSI protocol stack. Brief description of each module of figure 1 outlines the function modules in the simulator. Service Specific Convergence Sublayer (SSCS) is an implementation specific module and is the interface between 802.15.4 MAC and upper layers, which provides way to access all the MAC primitives. 802.15.4 MAC is the main module, implements all the 35 MAC Sublayer primitives.

The rest of this paper is organized in following sections: section 2, briefly discuss the IEEE 802.15.4 superframe structure. In section 3, mathematical model for pre and postsimulation analysis of TCL script is demonstrated. Section 4 presents algorithm. Performance metrics and experimental setup is given in section 5. Section 6 provides result for scenario taken into consideration, and this paper is concluded in section 7 . 


\section{IEEE 802.15.4 SUPERFRAME STRUCTURE}

IEEE 802.15.4 MAC protocol supports the beacon-enabled and no-beacon-enabled modes. In beacon-enabled mode, the access to the channel is managed through a superframe. The superframe is subdivided into a contention access period (CAP), contention free period (CFP) and inactive period. According to IEEE 802.15.4 standard, the active portion of each superframe consists of three parts: beacon, CAP and CFP, which is divided into 16 equal length slots. The length of each slot is equal to aBaseSlotDuration $\times 2^{\text {so }}$ symbols, where aBaseSlotDuration is equal to 60 symbols. The value of aBaseSuperframeDuration is fixed to 960 (16 x 60) symbols. In CAP, each node performs the CSMA/CA algorithm before transmitting data packet or control frame. Each node maintains three parameters: the number of backoffs (NB), contention window (CW), and back off exponent (BE).

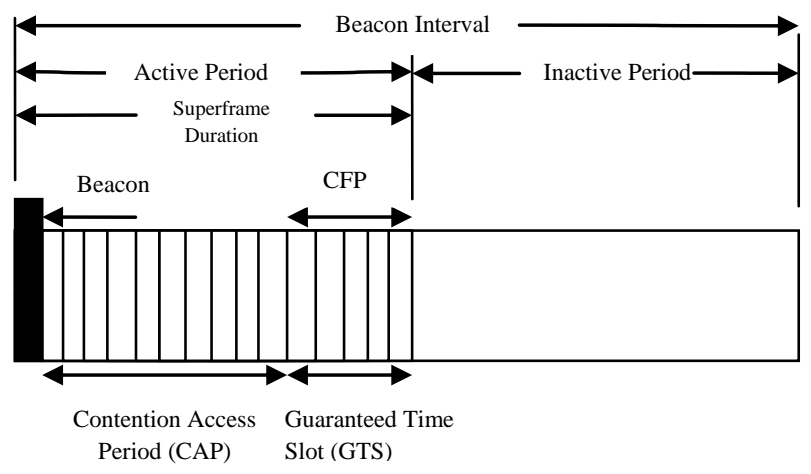

Figure 2 Superframe Structure

In beacon-enabled mode, each node employs two system parameters: beacon order (BO) and superframe order (SO). $\mathrm{BO}$ decides the length of beacon interval (BI), where

$\mathrm{BI}=$ aBaseSuperframeDuration $\times 2^{\mathrm{BO}}$ symbols and $0 \leq$ $\mathrm{BO} \leq 14$.

While SO decides the length of superframe duration (SD), where,

$\mathrm{SD}=$ aBaseSuperframeDuration $\mathrm{x} \quad 2^{\text {SO }}$ symbols and $0 \leq \mathrm{SO} \leq \mathrm{BO} \leq 14$.

In non-beacon-enabled mode, $\mathrm{BO}=\mathrm{SO}=15$, there are no regular beacons, but the coordinator may unicast beacons to soliciting device. The value of macSuperframeOrder shall be ignored if $\mathrm{BO}=15$.

Nodes in CAP use a slotted CSMA/CA to transmit their packets to contention for channel and CAP containing a number of GTSs that can be allocated by the PAN coordinator to specific nodes. Communication in non-beacon-enabled mode uses unslotted CSMA/CA for decentralized access.

\section{MATHEMATICAL MODEL FOR PRE-SIMULATION AND POST- SIMULATION}

\subsection{TCL Script to run 802.15.4 Scenario for pre-simulation analysis}

Consider a network containing nodes with fixed position for simulation process, given manually. NS2 generic script structure has been opted in order to analyze the TCL code for 802.15.4 scenario.
- $\quad$ Set the default parameters \{chan, prop, netif, mac, ifq, ll, rp, ant, ifqlen $\}$ where, chan = channel type, prop $=$ radio-propagation model, netif $=$ network interface type, $\mathrm{mac}=$ MAC layer type, ifq $=$ interface queue type, $11=$ link layer type, $\mathrm{rp}$ $=\mathrm{Ad}=$ hoc routing protocol type, ant $=$ antenna type, ifqlen $=$ max packet in interface queue type .

- Antenna setting \{Gt, Gr, height of antenna $\}$ where, $\mathrm{Gt}=$ gain of transmitting antenna, $\mathrm{Gr}=$ gain of receiving antenna.

- Physical layer setting \{freq, L, bandwidth, CPThresh, CSThresh, RXThresh $\}$ where, freq = working frequency band, $\mathrm{L}=$ system loss factor, CPThresh $=$ capture threshold $(\mathrm{db})$, CSThresh = carrier sense threshold $(\mathrm{W}), \mathrm{RXThresh}=$ receive power threshold (W).

- $\quad$ Set specified parameters for 802.15.4 MAC

- Create simulator object, topography object, General Operation Director (GOD), trace file to store information about nodes, their connections, traffic flow, and area under consideration.

- Implementation of the Service Specific Convergence Sublayer (SSCS)

To start a PAN Coordinator

\$node sscs startPANCoord <txBeacon $=1>$ $\langle$ beaconOrder $=4>\langle$ SuperframeOrder $=4\rangle$

To start a device or coordinator, \$node sscs startDevice $\langle$ isFFD=1> $\langle$ assoPermit=1> $<$ txBeacon $=0>$

$<$ SuperFrameOrder $=4>$ $<$ beaconOrder $=4>$

To start a GTS allocation mechanism \$node sscs startGTSDevice $\langle$ GTSCharacterics $=0 \mathrm{x} 1 \mathrm{c}\rangle \quad\langle$ txOption $=0 \mathrm{X} 02\rangle$ $<$ gtsPermit=1> $<$ SuperframeOrder $=4>$ $<$ BeaconOrder $=4>$

- Define stop time when simulation ends and tell nodes to reset which actually reset their internal network components. As well as flush out traces and close file descriptor.

\subsection{Post Simulation Analysis}

The output of the simulation process is as trace file. In first column, four type of packets s: send, r: receive, d: drop, f: forward, are indicated. Second column is time when event was started. The third column indicates the node number from. Fourth column used to indicate level like event generated at "MAC" level. Other than these four columns of trace file gives information about packet type, packet size, source and destination MAC address and time to send data etc.

Following shows part of NS2 trace file in the beacon-enabled mode $(\mathrm{BO}=\mathrm{SO}=4)$

s 0.787392000 _ 0 _ MAC --- 0 BCN 12 [0 ffffffff 00 0 [energy 0.999078 ei 0.000 es 0.000 et 0.000 er 0.001 ]

s 1.033152000 _ 0 - MAC --- 0 BCN 12 [0 ffffffff 00 0] [energy 0.998771 ei 0.000 es 0.000 et 0.001 er 0.001 ]

We set the values of $\mathrm{BO}=\mathrm{SO}=4$, so the value of $\mathrm{BI}$ is 15.36 x $2^{4}=245.76 \mathrm{~ms}$. From trace file, we can observe that node 0 sends BCN (beacon) at every $245.76 \mathrm{~ms}$ (1.0331520000.787392000 ). 
s $1.501920000 \_1$ _ MAC --- 0 CM1 8 [0 ffffffff 20 0] [energy 0.998714 ei 0.000 es 0.000 et 0.000 er 0.001 ]

Data transmission can from device to the coordinator or from the coordinator to the device. Following shows the node 1 sends an association request (CM1) node 0 .

r 1.502368017 _0_ MAC --- 0 CM1 8 [0 ffffffff 20 ] [energy 0.998675 ei 0.000 es 0.000 et 0.000 er 0.001$]$

\section{ALGORITHM}

First part contains Tool Command Language (TCL) file simulation in NS2 and second part consists of algorithm for performance evaluation of trace file which is output of simulated TCL file.

\section{A. Algorithm for TCL File}

Step 1: Start

Step 2: Create simulator instance

Step 3: Setup trace support by opening file out.tr and out.nam in writing mode and call the procedure trace-all.

Step 4: Design a topology object.

Step 5: Make topography into grids.

Step 6: Make an object of General Operations Director (GOD)

Step 7: Configure nodes using node configuration API.

Step 8: Design scenario with fixed positions of nodes.

Step 9: Setup traffic flow between nodes.

Step 10: Set stop simulation with overall reset network scenario, flush out traces and closed trace file.

Step 11: Run the simulation.

Step 12: Stop.

\section{B. Algorithm for performance evaluations}

Step 1: Start

Step 2: Collect all the parameters from trace file.

Step 3: Using AWK scripts of various performance metrics parameters, insert the formula and generates the results.

Step 4: Generate graphs from results.

Step 5: Stop

\section{Pseudo code for association procedure}

Channel scan

if coordinators not found association fail

else if no coordinators permit association association fail else

select a proper coordinator

send association request to the coordinator

wait for ACK

if ACK not received association fail

else

send data request to the coordinator

wait for ACK

if ACK not received

association fail

else

wait for association response

if association response not received

association fail

else if association not granted

association fail

else

association succeed

send GTS request to the coordinator

wait for ACK

if ACK not received

GTS fail

else

wait for $\mathrm{BCN}$

if $\mathrm{BCN}$ not received

GTS fail

else

GTS succeeds

\section{Energy consumption}

- Energy consumption of coordinator node: It includes the energy consumption for idle state, transmitting beacon, receiving data at CSMA/CA in $\mathrm{CAP}$, receiving request for GTS allocation, receiving data at GTS in CFP, and transmitting ACK for receiving successful packet, both in CAP in CFP.

- Energy consumption of device node: It includes energy consumption for back-off, CCA transmission, data packet transmission in CAP, GTS request for CFP, data packet transmission in CFP, deferred transmission waiting time, idle, receiving beacon, receiving ACK for both CAP and CFP successful packet transmission.

\section{PERFORMANCE METRICS AND} EXPERIMENTAL SETUP

\section{A. Performance Metrics}

- Packet delivery ratio: The ratio of packet successfully received to packets sent in MAC sublayer. This metric includes both transmitted packets.

- Energy consumption: The coordinator consumes energy in transmitting beacon and ACK packet, receives data packet and scanning the channel. 
- Duty cycle: The ratio of the active duration, including transmission, reception and carrier sense time of a transceiver to the whole session duration.

- Successful association rate: The ratio of devices successfully associated with coordinator to the total devices trying to associate with coordinator.

- Orphaning rate: It is the ratio of devices that are in beacon enabled mode and keep tracking beacons. It is not applicable for non-beaconed mode.

- End-to-end delay: It is the time elapsed by a successful received packet from source to destination node, which includes route discovery delay, propagation time, data transfer time and in between queuing delays.

\section{B. Experimental setup}

802.15.4 performance through NS2 simulator has been performed. A bounded region of 50x50 sqm, has been taken with fixed position of nodes. The sensing range of nodes has been set upto 40 meters. Constant bit rate (CBR) traffic, Poisson traffic with packet size of 70 bytes at the rate of 250 kbps and file transfer protocol (FTP) traffic with packet size of 50 bytes are used.

\section{Simulation parameters}

For simulation, the Carrier sense threshold (CSThresh), Receiving threshold (RxThresh) and Carrier power threshold (CPThresh) has been set. CSThresh is set to determine whether frame is detected by the receiver or not. If the signal strength of the frame is less than the CSThresh, the frame is not passed on to MAC but is discarded at the PHY. RxThresh determines if the frame is received correctly or not. If the signal strength of the received frame is below the RxThresh is marked as an error and passed on to MAC where it is discarded. CPThresh determines if a frame is received correctly or not. If a node receives multiple frames simultaneously, it calculates the ratio of strongest frame signal to the signal strength and compares with the CPThresh, if the calculated ratio is larger than CPThresh, frame is received correctly and all other frames are discarded or said to be collided.

Table 1 Simulation Parameters

\begin{tabular}{|c|c|}
\hline Component & Type \\
\hline Channel Type & $\begin{array}{c}\text { Channel/Wireless } \\
\text { Channel }\end{array}$ \\
\hline Antenna model & Omni directional \\
\hline Radio Propagation Model & Two Ray Ground \\
\hline Network Interface Type & Phy/Wireless Phy \\
\hline MAC layer Protocol & 802.15 .4 \\
\hline PHY layer Protocol & 802.15 .4 \\
\hline Interface Queue Type & Queue/DropTail/PriQueu \\
& e \\
\hline Topology Size & $50 \times 50$ \\
\hline Frequency used & $2.4 \mathrm{GHz}$ \\
\hline
\end{tabular}

\section{RESULT ANALYSIS}

Figure 3 and figure 4 shows the packet delivery ratio with varying traffic load, beacon order and superframe order. Smaller BO results in high duty cycle, achieve higher bandwidth, which makes the throughput better by making more successful transmission in network.

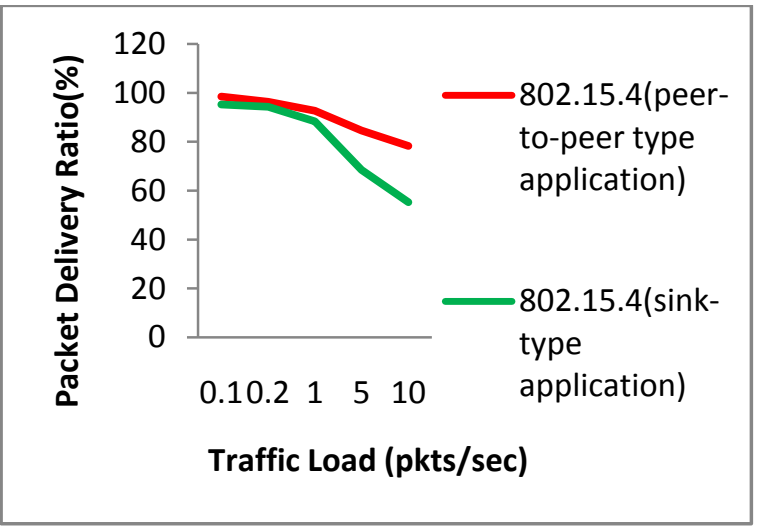

Figure 3 Packet Delivery Ratio Vs Traffic load

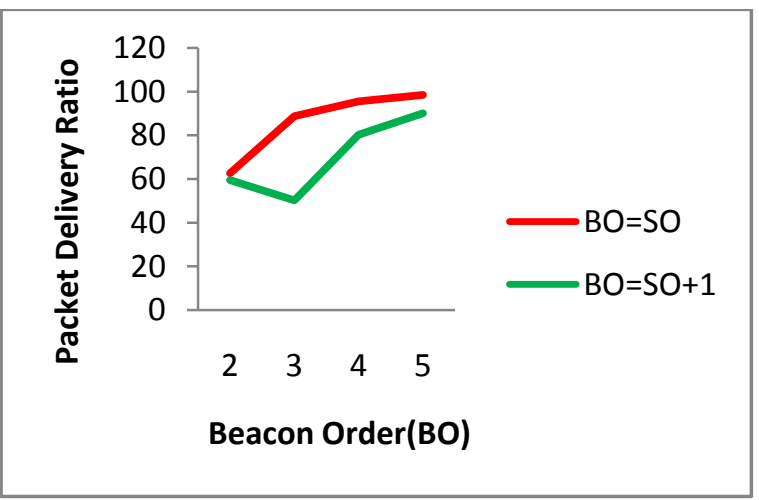

Figure 4 Packet Delivery Ratio Vs BO

Figure 5 and figure 6 shows energy consumption in three modes (transmit, receive and idle) is plotted with varying $\mathrm{BO}$ and SO. With decreasing BO, energy consumption in transmitting and receiving mode is increasing and decreasing in case of idle mode while with increasing SO, energy consumption in transmitting and receiving mode is increasing and decreasing in case of idle mode. This is due to the fact that with increase in SO and decrease in BO, duty cycle increases and reduces the idle period that results in spending more time during transmission and reception and thus idle time is decreased.

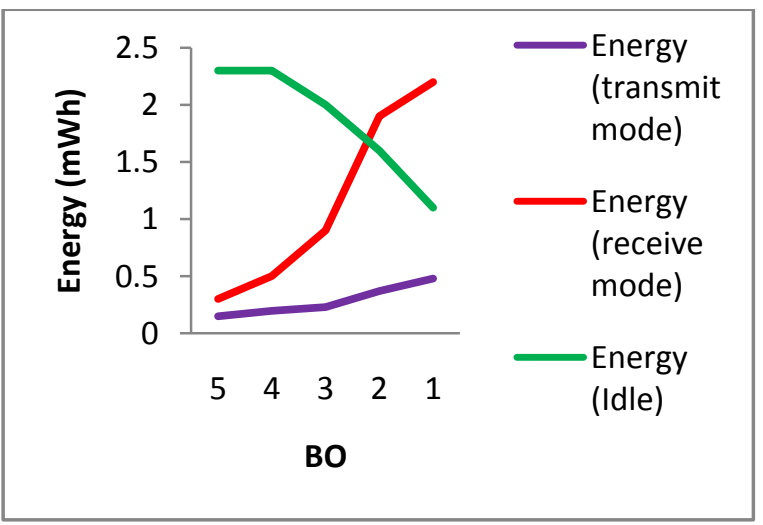

Figure 5 Energy Vs BO 


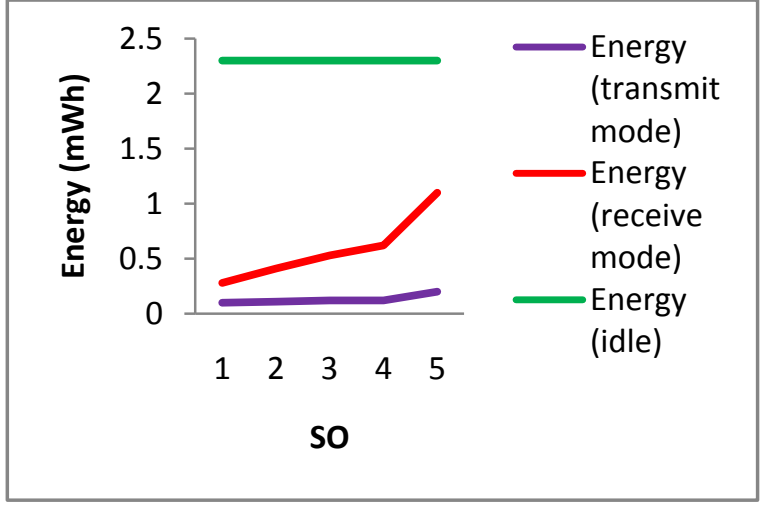

Figure 6 Energy Vs SO

Figure 7 shows the average end-to-end delay with varying $\mathrm{BO}$ and SO. Larger BO values causes longer inactive period, which may experience a longer sleep delay for the buffered packets and vice versa for smaller SO.

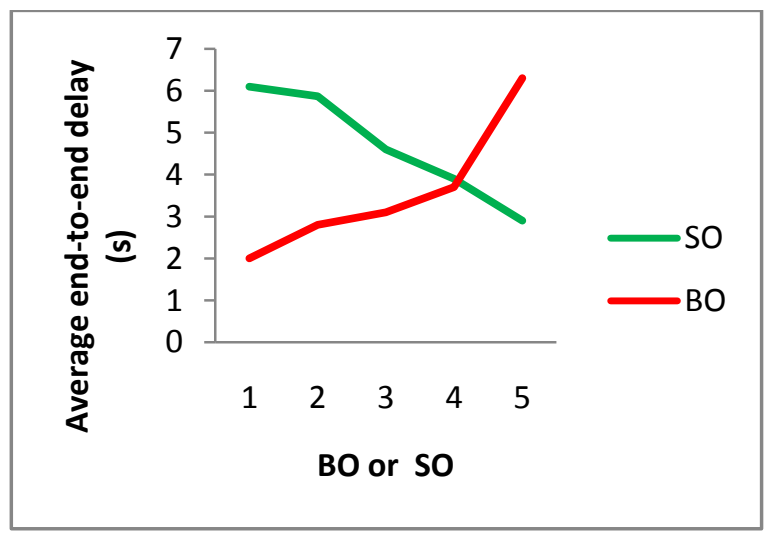

Figure 7 Average end-to-end delay Vs BO or SO

\section{CONCLUSION}

Mathematical model of 802.15.4 are validated and simulated to analyze the pre-simulation and post-simulation using trace file generated, to check performance of superframe duration by setting the value of appropriate $\mathrm{BO}$, SO with different traffic types. Duty cycle is the main parameter that is influenced by varying $\mathrm{BO}$ and SO. Better performance of 802.15.4 network is seen with increasing the duty cycle. Adjustable length of slots of superframe duration effectively improves the IEEE 802.15.4 medium access control layer in both CAP and CFP.

\section{REFERENCES}

[1] IEEE 802.15.4, part 15.4: wireless medium access control (MAC) and physical layer (PHY) specifications for low-rate wireless personal area networks (WPANs), IEEE standard for information technology, 2006.

[2] Network Simulator, http://www.isi.edu/nsnam/ns

[3] K Ashrafuzzaman, K Sup Kwak, on the performance analysis of the contention access period of IEEE 802.15.4. IEEE Communication Lett 15, 9 (2011)

[4] AN Alvi, SS Naqvi, SH Bouk, N Javaid, U Qasim, ZA Khan, Evaluation of slotted CSMA/CA of IEEE 802.15.4 (Seventh International Conference on Broadband, Wireless Computing, Communication and Applications,, Canada, 2012)
[5] T-R Park, T-H Kim, J-Y Choi, W-H Kwon, Throughput and energy consumption analysis of IEEE 802.15.4 slotted CSMA/CA. IEEE Electron Letter 41 (18), 2005.

[6] TJ Jee, HR Lee, MY Chung, MAC throughput limit analysis of slotted CSMA/CA in IEEE 802.15.4 WPAN. IEEE Communication Letter 10 (7), 2006.

[7] Y Zhang, F Shu, Packet Size Optimization for Goodput and Energy Efficiency Enhancement in Slotted IEEE 802.15.4 networks. IEEE Wireless Communications Networking Conference, 2009.

[8] S Pollin, M Ergen, S Ergen, B Bougard, L Der Perre, I Moerman, A Bahai, P Varaiya, F Catthoor, Performance analysis of slotted carrier sense IEEE 802.15.4 medium access layer. IEEE Transaction Wireless Communication 7 (9), 2008

[9] J He, Z Tang, HH Chen, Q Zhang, An accurate and scalable analytical model for IEEE 802.15.4 slotted CSMA/CA networks. IEEE Wireless Comm 8(1), 2009.

[10] C Buratti, Performance analysis of IEEE 802.15.4 beacon enabled mode. IEEE Trans.Veh. Technol 59, 2010 .

[11] Z Xiap, C He, L Jiang, An analytical model for IEEE 802.15.4 with sleep mode based on time-varying queue, in IEEE International Conference on Communications (ICC), Kyoto, Japan, 2011.

[12] Z Tao, S Panwar, D Gu, J Zhang, Performance analysis and a proposed improvement for the IEEE 802.15.4 contention access period. IEEE WCNC 4, 2006.

[13] B-H Lee, M Udin Harun Al Rasyid, H-K Wu, Anlysis of superframe adjustment and beacon transmission for IEEE 802.15.4 Cluster tree Networks. EURASHIP Journal on Wireless Communication and Networking, 2012.

[14] M Maralo, S Busanelli, G Ferrari, Markov chain-based performance analysis of multihop IEEE 802.15.4 wireless networks. 66. 2009

[15] A Koubaa, M Aves, E Tovar, GTS allocation analysis in IEEE 802.15.4 for real-time wireless sensor networks, International Parallel and Distributed Processing Symposium, IEEE, 2006.

[16] P Park, C Fischione, K Johansson, Performance analysis of GTS allocation in beacon enabled IEEE 802.15.4, in 6th Annual IEEE Communications Society Conference on Sensor, Mesh and AdHoc Communications and Networks, 2009.

[17] S-T Sheu, YY Shih, WT Lee, CSMA/CA protocol for IEEE 802.15.4 WPANs, IEEE Trans. Veh. Technology 58(3), 2009.

[18] A Koubaa, M Aves, E Tovar, A Cunha, An implicit GTS allocation mechanism in IEEE 802.15.4 for timesensitive wireless sensor networks: theory and practice, Springer Real-Time systems, 39, 2008.

[19] L-C Ko, Z-T Chou, A Novel Multi-beacon superframe structure with greedy GTS Allocation for IEEE 802.15.4 Wireless PANs. IEEE Wireless Communications and Networking Conference (WCNC), 2007 